\title{
A Parameter Perturbation Homotopy Continuation Method for Solving Fixed Point Problems with Both Inequality and Equality Constraints
}

\author{
Menglong Su, ${ }^{1,2}$ Yufeng Shang, and Wenzhuang $\mathrm{Zhu}^{4}$ \\ ${ }^{1}$ School of Mathematics, Luoyang Normal University, Luoyang 471934, China \\ ${ }^{2}$ Key Laboratory of Symbolic Computation and Knowledge Engineering of Ministry of Education, Jilin University, \\ Changchun 130012, China \\ ${ }^{3}$ Section of Mathematics, Aviation University of Air force, Changchun 130022, China \\ ${ }^{4}$ School of Mathematics, Nankai University, Tianjin 300071, China
}

Correspondence should be addressed to Menglong Su; sumenglongjlu@163.com

Received 9 December 2016; Accepted 23 January 2017; Published 13 February 2017

Academic Editor: Maria L. Gandarias

Copyright (c) 2017 Menglong Su et al. This is an open access article distributed under the Creative Commons Attribution License, which permits unrestricted use, distribution, and reproduction in any medium, provided the original work is properly cited.

In this paper, we propose a parameter perturbation homotopy continuation method for solving fixed point problems on more general nonconvex sets with both inequality and equality constraints. By adopting appropriate techniques, we make the initial points not certainly in the set consisting of the equality constraints. This point can improve the computational efficiency greatly when the equality constraints are complex. In addition, we also weaken the assumptions of the previous results in the literature so that the method proposed in this paper can be applied to solve fixed point problems in more general nonconvex sets. Under suitable conditions, we obtain the global convergence of this homotopy continuation method. Moreover, we provide several numerical examples to illustrate the results of this paper.

\section{Introduction}

Fixed point theorems have been widely applied to many areas such as mechanics, physics, transportation, control, economics, differential equations, and optimization. Particularly the algorithm construction of computing a fixed point has attracted much attention and lots of results have appeared. The results of related research mentioned above are found in the literature (see [1-10], etc. and the references therein). Although the homotopy method has become a popular tool in dealing with fixed point problems (see [1115], etc. and the references therein), this method still requires certain convexity assumptions. These convexity assumptions prevent the homotopy method from being extended to provide a constructive proof of the general Brouwer fixed point theorem, which does not require the convexity of the subsets in $R^{n}$. Recently, on a class of nonconvex subset that satisfies the normal cone condition, by introducing the ideas of Karmarkar's interior point method into the homotopy method, Yu and Lin [16] proposed a combined homotopy interior point method to give a constructive proof of the general Brouwer fixed point theorem and hence solve fixed point problems numerically. In [17], by introducing $C^{2}$ mappings $\alpha(x)=\left(\alpha_{1}(x), \ldots, \alpha_{m}(x)\right) \in R^{n \times m}$ and $\beta(x)=$ $\left(\beta_{1}(x), \ldots, \beta_{l}(x)\right) \in R^{n \times l}$, we further extended the results in [16] to more general nonconvex sets with both inequality and equality constraint functions. In 2013, by introducing $C^{2}$ functions $\eta_{i}\left(x, u_{i}\right) \in R^{n}, i=1, \ldots, m$, and $\zeta_{j}\left(x, v_{j}\right) \in R^{n}$, $j=1, \ldots, l$, Zhu et al. [18] further extended the results in [17] to more general nonconvex sets. It should be pointed out that, in [18], the initial point $x^{(0)}$ must satisfy the equation $h(x)=$ 0 . In fact, it is difficult to select such an initial point when the constraint function $h(x)$ is complex. This point reduces the computational efficiency of the homotopy interior point 
method greatly. In this paper, we propose a parameter perturbation homotopy continuation method for solving fixed point problems with both inequality and equality constraints. By using this new homotopy continuation method, we can not only remove the assumption that the matrix $\nabla_{z}\left(\sum_{j=1}^{l} \zeta\left(x, z_{j}\right)\right)$ is of full row rank in [18] but also can make the initial point $x^{(0)}$ only satisfy $g(x)<0$, without satisfying the equation $h(x)=0$. Moreover, we mainly extend the results in [19, 20 ] to more general nonconvex sets and provide a practical perturbation for the inequality constraints compared with the results in [20]. Under commonly used conditions in the literature, we obtain the global convergence of the parameter perturbation continuation method. Moreover, we provide several numerical examples to illustrate the results of this paper.

This paper is organized as follows. Section 2 contains the basic definitions and the required preliminary materials widely used in what follows. Section 3 is the main part, which exhibits a convergence proof of the parameter perturbation continuation method. Section 4 presents the use of the reduced predictor-corrector algorithms given by Allgower and Georg [11] to compute a number of experimental examples, which illustrate the results of this paper.

\section{Preliminaries}

In this section, we need the following notations: $\Omega=\{x \in$ $\left.R^{n}: g_{i}(x) \leq 0, i=1, \ldots, m, h_{j}(x)=0, j=1, \ldots, l\right\}, \Omega^{0}=$ $\left\{x \in R^{n}: g_{i}(x)<0, i=1, \ldots, m, h_{j}(x)=0, j=1, \ldots, l\right\}$, $R_{+}^{m}=\left\{x \in R^{m}: x \geq 0\right\}, R_{++}^{m}=\left\{x \in R^{m}: x>0\right\}$, and $B(x)=\left\{i \in\{1, \ldots, m\}: g_{i}(x)=0\right\}, \Omega_{g}=\left\{x \in R^{n}: g_{i}(x) \leq\right.$ $0, i=1, \ldots, m\}, \Omega_{g}^{0}=\left\{x \in R^{n}: g_{i}(x)<0, i=1, \ldots, m\right\}$, and $\partial \Omega_{g}=\Omega_{g} \backslash \Omega_{g}^{0}$.

In [18], the initial point $x^{(0)}$ is confined in the interior of $\Omega^{0}$; thus $x^{(0)}$ must satisfy $h\left(x^{(0)}\right)=0$ as well as $g\left(x^{(0)}\right)<0$. Once the equality constraint function $h(x)$ is complex, it is difficult to select such an initial point $x^{(0)}$. This point may reduce the computational efficiency of the homotopy interior point method greatly. In this paper, we attempt to construct a new homotopy equation, which not only makes us remove the assumption that $\nabla_{z}\left(\sum_{j=1}^{l} \zeta\left(x, z_{j}\right)\right)$ is of full row rank in [18] but also makes the initial point $x^{(0)}$ only satisfy $g(x)<0$, without satisfying the equation $h(x)=0$. To this end, we construct a new homotopy equation as follows:

$$
H\left(w, w^{(0)}, \lambda\right)=\left(\begin{array}{c}
(1-\lambda)(x-\Phi(x))+\sum_{i=1}^{m} \eta_{i}\left(x,(1-\lambda) y_{i}\right)+\sum_{j=1}^{l} \zeta_{j}\left(x,(1-\lambda) z_{j}\right)+\lambda\left(x-x^{(0)}\right) \\
h(x)-\lambda z \\
y_{1}\left(g_{1}\left(A_{1}(\lambda) x+b_{1}(\lambda)\right)+\lambda c_{1}\right)-\lambda y_{1}^{(0)}\left(g_{1}\left(A(1) x^{(0)}+b_{1}(1)\right)+c_{1}\right) \\
\vdots \\
y_{m}\left(g_{m}\left(A_{m}(\lambda) x+b_{m}(\lambda)\right)+\lambda c_{m}\right)-\lambda y_{m}^{(0)}\left(g_{m}\left(A(1) x^{(0)}+b_{m}(1)\right)+c_{m}\right)
\end{array}\right)=0
$$

where $w=(x, y, z) \in R^{n} \times R_{+}^{m} \times R^{l}, h(x)=\left(h_{1}(x)\right.$, $\left.\ldots, h_{l}(x)\right)^{T} \in R^{l}$, and $A_{i}(\lambda) \in R^{n \times n}$ and $b_{i}(\lambda) \in R^{n}, i=$ $1, \ldots, m$. Besides, $c_{i} \in R^{1}, i=1, \ldots, m$, are constants. Set $\Omega(\lambda)=\left\{x \in R^{n}: g_{i}\left(A_{i}(\lambda) x+b_{i}(\lambda)\right)+\lambda c_{i} \leq 0, i=\right.$ $1, \ldots, m, h(x)=0\}, \Omega^{0}(\lambda)=\left\{x \in R^{n}: g_{i}\left(A_{i}(\lambda) x+\right.\right.$ $\left.\left.b_{i}(\lambda)\right)+\lambda c_{i}<0, i=1, \ldots, m, h(x)=0\right\}, I(x, \lambda)=\{i \in$ $\left.\{1, \ldots, m\}: g_{i}\left(A_{i}(\lambda) x+b_{i}(\lambda)\right)+\lambda c_{i}=0\right\}, \Omega_{g}(\lambda)=\{x \in$ $\left.R^{n}: g_{i}\left(A_{i}(\lambda) x+b_{i}(\lambda)\right)+\lambda c_{i} \leq 0, i=1, \ldots, m\right\}, \Omega_{g}^{0}(\lambda)=$ $\left\{x \in R^{n}: g_{i}\left(A_{i}(\lambda) x+b_{i}(\lambda)\right)+\lambda c_{i}<0, i=1, \ldots, m\right\}$, and $\partial \Omega_{g}(\lambda)=\Omega_{g}(\lambda) \backslash \Omega_{g}^{0}(\lambda)$.

Next, we make the following assumptions:

$\left(A_{1}\right) \Omega^{0}$ is nonempty and $\Omega_{g}(\lambda)$ is bounded.

$\left(A_{2}\right) \eta_{i}(x, 0)=0, i=1, \ldots, m$, and $\zeta_{j}(x, 0)=0, j=$ $1, \ldots, l$; besides, for any $x \in \Omega_{g}(\lambda)$ and for any $u \in R_{+}^{m}$ and $v \in R^{l}$, if $\|(u, v)\| \rightarrow \infty$, then

$$
\left\|\sum_{i \in I(x, \lambda)} \eta_{i}\left(x, u_{i}\right)+\sum_{j=1}^{l} \zeta\left(x, v_{j}\right)\right\| \longrightarrow \infty
$$

\section{$\left(A_{3}\right) \forall x \in \Omega$, if}

$$
\sum_{i \in B(x)} \eta_{i}\left(x, u_{i}\right)+\sum_{j=1}^{l} \zeta\left(x, v_{j}\right)=0, \quad u_{i} \geq 0
$$

then $u_{i}=0, \forall i \in B(x)$, and $v_{j}=0, j=1, \ldots, l$. In addition, for any $x \in \Omega_{g}(\lambda), \nabla h(x)$ is a matrix of full column rank.

$\left(A_{4}\right) \forall x \in \Omega$, we have

$$
\begin{aligned}
& \left\{x+\sum_{i \in B(x)} \eta_{i}\left(x, u_{i}\right)+\sum_{j=1}^{l} \zeta\left(x, v_{j}\right): u_{i} \geq 0, i \in B(x)\right\} \\
& \cap X=\{x\} .
\end{aligned}
$$

Besides, $\forall x \in \partial \Omega_{g}(\lambda)$, we have

$$
\begin{aligned}
& \left\{x+\sum_{i \in I(x, \lambda)} \eta_{i}\left(x, u_{i}\right): u_{i} \geq 0, i \in I(x, \lambda)\right\} \cap \Omega_{g}(\lambda) \\
& =\{x\} .
\end{aligned}
$$




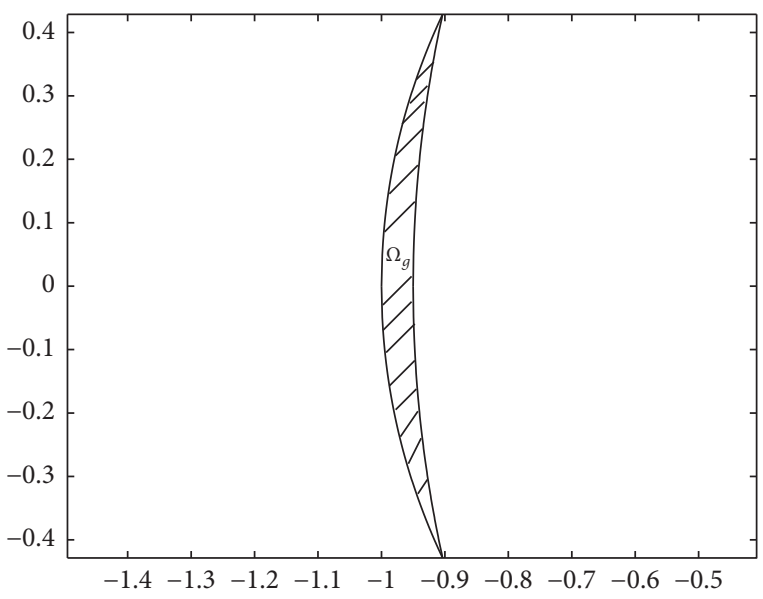

FIgURE 1: The original feasible set $\Omega_{g}$ of Example 1 .

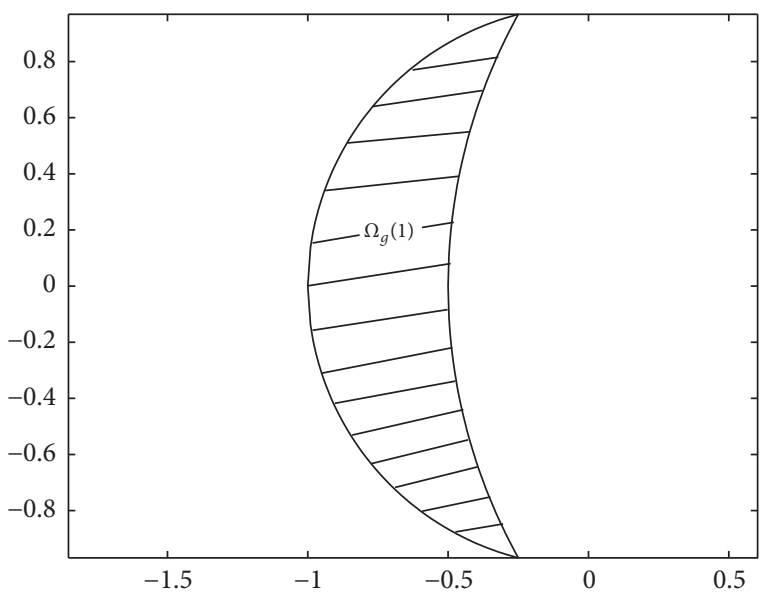

Figure 2: The perturbated feasible set $\Omega_{g}(1)$ of Example 1 .

Example 1. To find a fixed point of self-mapping $\Phi(x)=$ $\left(x_{1},-x_{2}\right)^{T}$ in $\Omega_{g}=\left\{\left(x_{1}, x_{2}\right) \in R^{2}: x_{1}^{2}+x_{2}^{2} \leq 1,\left(x_{1}-\right.\right.$ $\left.1.05)^{2}+x_{2}^{2} \geq 4\right\}$, in this example, set $g_{1}(x)=x_{1}^{2}+x_{2}^{2}-1$ and $g_{2}(x)=-\left(x_{1}-1.05\right)^{2}-x_{2}^{2}+4$; the original feasible set $\Omega_{g}$ (See Figure 1) is so small that we cannot find an initial point easily. However, set $A_{1}(\lambda)=I_{2 \times 2}, A_{2}(\lambda)=I_{2 \times 2}, b_{1}(\lambda)=(0,0)^{T}$, $b_{2}(\lambda)=(-0.45 \lambda, 0)^{T}, c_{1}=0$, and $c_{2}=0$; then

$$
\begin{aligned}
& g_{1}\left(A_{1}(\lambda) x+b_{1}(\lambda)\right)+\lambda c_{1}=g_{1}(x), \\
& g_{2}\left(A_{2}(\lambda) x+b_{2}(\lambda)\right)+\lambda c_{2} \\
& \quad=-\left(x_{1}-1.05-0.45 \lambda\right)^{2}-x_{2}^{2}+4 .
\end{aligned}
$$

When $\lambda=1$, the perturbated feasible set $\Omega_{g}(1)$ (see Figure 2) becomes big enough to find an initial point more easily than before.
For given $w^{(0)}$, rewrite $H\left(w, w^{(0)}, \lambda\right)$ as $H_{w^{(0)}}(w, \lambda)$. The zero-point set of $H_{w^{(0)}}$ is

$$
\begin{aligned}
& H_{w^{(0)}}^{-1}(0)=\left\{(w, \lambda) \in \Omega_{g}(\lambda) \times R_{+}^{m} \times R^{l}\right. \\
& \left.\quad \times(0,1]: H_{w^{(0)}}(w, \lambda)=0\right\} .
\end{aligned}
$$

Remark 2. In [18], to guarantee that the homotopy smooth curve is diffeomorphic to a unit interval $(0,1]$, we need to prove that the matrix

$$
\begin{aligned}
& \frac{\partial \widetilde{H}_{w^{(0)}}\left(w^{(0)}, 1\right)}{\partial w} \\
& =\left(\begin{array}{ccc}
I & 0 & \nabla_{z}\left(\sum_{j=1}^{l} \zeta\left(x^{(0)}, z_{j}^{(0)}\right)\right) \\
\nabla h\left(x^{(0)}\right)^{T} & 0 & 0 \\
Y^{(0)} \nabla g\left(x^{(0)}\right)^{T} & \operatorname{diag}\left(g\left(x^{(0)}\right)\right) & 0
\end{array}\right)
\end{aligned}
$$

is nonsingular, where $\widetilde{H}$ is the homotopy in [18]. This requires that the matrix $\nabla_{z}\left(\sum_{j=1}^{l} \zeta\left(x, z_{j}\right)\right)$ be of full row rank. In new homotopy (1), the matrix

$$
\frac{\partial H_{w^{(0)}}\left(w^{(0)}, 1\right)}{\partial w}=\left(\begin{array}{ccc}
I & 0 & 0 \\
\nabla h\left(x^{(0)}\right)^{T} & 0 & -I \\
A & B & 0
\end{array}\right)
$$

is nonsingular without requiring the assumption that the matrix $\nabla_{z}\left(\sum_{j=1}^{l} \zeta\left(x, z_{j}\right)\right)$ is of full row rank, where

$$
\begin{aligned}
A= & Y^{(0)}\left(\nabla_{x} g_{1}\left(A_{1}(1) x^{(0)}+b_{1}(1)\right), \ldots,\right. \\
& \left.\nabla_{x} g_{m}\left(A_{m}(1) x^{(0)}+b_{m}(1)\right)\right)^{T}, \\
B= & \operatorname{diag}\left(g_{1}\left(A_{1}(1) x^{(0)}+b_{1}(1)\right)\right. \\
& \left.+c_{1}, \ldots, g_{m}\left(A_{m}(1) x^{(0)}+b_{m}(1)\right)+c_{m}\right) .
\end{aligned}
$$

Remark 3. When $\lambda=1$, the homotopy in [18] becomes

$$
\begin{aligned}
\sum_{j=1}^{l} \zeta_{j}\left(x, z_{j}\right)+x-x^{(0)} & =0, \\
h(x) & =0, \\
Y g(x)-Y^{(0)} g\left(x^{(0)}\right) & =0 .
\end{aligned}
$$

It is easy to see that the initial point $x^{(0)}$ must satisfy $h(x)=0$ as well as $g(x) \leq 0$. This point makes the choice of initial points become difficult when the function $h(x)$ is complex. However, from new homotopy (1), when $\lambda=1$, we have

$$
\begin{aligned}
& x-x^{(0)}=0, \\
& h(x)-z=0, \\
& y_{i}\left(g_{i}\left(A_{i}(1) x+b_{i}(1)\right)+c_{i}\right) \\
& \quad-y_{i}^{(0)}\left(g_{i}\left(A_{i}(1) x^{(0)}+b_{i}(1)\right)+c_{i}\right)=0, \\
& \quad i=1, \ldots, m .
\end{aligned}
$$


From (12), we conclude that $x^{(0)}$ only needs to satisfy $g_{i}\left(A_{i}(1) x+b_{i}(1)\right)+c_{i} \leq 0, i=1, \ldots, m$, without satisfying the equation $h(x)=0$.

In the following, we recall some basic definitions and results from differential topology, which will be used in our main result of this paper.

The inverse image theorem (see [21]) tells us that if 0 is a regular value of the map $H_{w^{(0)}}$, then $H_{w^{(0)}}^{-1}(0)$ consists of some smooth curves. And the regularity of $H_{w^{(0)}}$ can be obtained by the following lemma.

Lemma 4 (parameterized Sard's theorem). Let $V \subset R^{n}$ and $U \subset R^{m}$ be open sets, and let $\Phi: V \times U \rightarrow R^{k}$ be a $C^{r}$ map, where $r>\max \{0, m-k\}$. If $0 \in R^{k}$ is a regular value of $\Phi$, then, for almost all $a \in V, 0$ is a regular value of $\Phi_{a} \equiv \Phi(a, \cdot)$.

\section{Convergence Analysis}

In this section, we are devoted to giving the global convergence analysis of the parameter perturbation homotopy continuation method.

Lemma 5. Let $H$ be defined as in (1), let $g_{i}(x), i=1, \ldots, m$, and $h_{j}(x), j=1, \ldots, l$, be $C^{3}$ functions, let assumptions $\left(A_{1}\right)$ $\left(A_{4}\right)$ hold, and let $\eta_{i}\left(x, u_{i}\right) \in R^{n}, i=1, \ldots, m$, and $\zeta_{j}\left(x, v_{j}\right) \in$ $R^{n}, j=1, \ldots, l$, be $C^{2}$ functions. Then, for almost all $x^{0} \in$ $\Omega_{g}^{0}(1), 0$ is a regular value of map $H_{w^{(0)}}: R^{n} \times R_{+}^{m} \times R^{l} \times(0,1] \rightarrow$ $R^{n+m+l}$, and there exists a $C^{1}$ curve $(w(s), \lambda(s))$ of dimension 1 such that

$$
\begin{aligned}
H\left(w(s), w^{(0)}, \lambda(s)\right) & =0, \\
(w(0), \lambda(0)) & =\left(w^{(0)}, 1\right) .
\end{aligned}
$$

Proof. When $x^{(0)}$ is considered as a variable, let the Jacobian matrix of $H_{w^{(0)}}(w, \lambda)$ be denoted by $D H_{w^{(0)}}(w, \lambda)$, for any $\lambda \epsilon$ $(0,1]$ :

$$
\frac{\partial H_{w^{(0)}}(w, \lambda)}{\partial\left(x, x^{(0)}, y^{(0)}\right)}=\left(\begin{array}{ccc}
C & -\lambda I & 0 \\
\nabla h(x)^{T} & 0 & 0 \\
D & -\lambda A(1) A & -\lambda B
\end{array}\right),
$$

where

$$
\begin{aligned}
C= & (1-\lambda)(I-\nabla \Phi(x))+\nabla_{x}\left(\sum_{i=1}^{m} \eta_{i}\left(x,(1-\lambda) y_{i}\right)\right) \\
& +\nabla_{x}\left(\sum_{j=1}^{l} \zeta_{j}\left(x,(1-\lambda) z_{j}\right)\right)+\lambda I \\
D & =Y\left(\nabla_{x} g_{1}\left(A_{1}(\lambda) x+b_{1}(\lambda)\right), \ldots,\right. \\
& \left.\nabla_{x} g_{m}\left(A_{m}(\lambda) x+b_{m}(\lambda)\right)\right)^{T} .
\end{aligned}
$$

Because $g_{i}\left(A_{i}(1) x^{(0)}+b_{i}(1)\right)+c_{i}<0, i=1, \ldots, m$, and $\nabla h(x)$ is a matrix of full column rank, then $\partial H_{w^{(0)}}(w, \lambda) / \partial\left(x, x^{(0)}, y^{(0)}\right)$ is a matrix of full row rank. Moreover, it is easy to show that $D H_{w^{(0)}}(w, \lambda)$ is of full row rank and thus 0 is a regular value of $H_{w^{(0)}}(w, \lambda)$. By the parameterized Sard's theorem, for almost all $x^{(0)} \in \Omega_{g}^{0}(1), 0$ is a regular value of the map $H_{w^{(0)}}: R^{n} \times R_{+}^{m} \times R^{l} \times(0,1] \rightarrow R^{n+m+l}$. By the inverse image theorem, $H_{w^{(0)}}^{-1}(0)$ consists of some smooth curves. Because $H_{w^{(0)}}(w, 1)=0$ has a unique solution $w^{(0)}=$ $\left(x^{(0)}, y^{(0)}, h\left(x^{(0)}\right)\right)$, then there exists a $C^{1}$ curve $(w(s), \lambda(s))$ (denoted by $\left.\Gamma_{w^{(0)}}\right)$ of dimension 1 such that

$$
\begin{aligned}
H\left(w(s), w^{(0)}, \lambda(s)\right) & =0, \\
(w(0), \lambda(0)) & =\left(w^{(0)}, 1\right) .
\end{aligned}
$$

Lemma 6. Let $H$ be defined as in (1), let $g_{i}(x), i=1, \ldots, m$, and $h_{j}(x), j=1, \ldots, l$, be $C^{3}$ functions, let assumptions $\left(A_{1}\right)$ $\left(A_{4}\right)$ hold, and let $\eta_{i}\left(x, u_{i}\right) \in R^{n}, i=1, \ldots, m$, and $\zeta_{j}\left(x, v_{j}\right) \in$ $R^{n}, j=1, \ldots, l$, be $C^{2}$ functions. Then, for almost all $x^{(0)} \in$ $\Omega_{g}^{0}(1), \Gamma_{w^{(0)}}$ is a bounded curve.

Proof. Assume that $\Gamma_{w^{(0)}}$ is an unbounded curve. Then there exists a sequence of points $\left\{\left(w^{(k)}, \lambda_{k}\right)\right\} \subset \Gamma_{w^{(0)}}$ such that $\left\|\left(w^{(k)}, \lambda_{k}\right)\right\| \rightarrow \infty$. Because $\Omega_{g}(\lambda)$ and $(0,1]$ are bounded, hence there exists a subsequence of points (denoted also by $\left.\left\{\left(w^{(k)}, \lambda_{k}\right)\right\}\right)$ such that $x^{(k)} \rightarrow x^{*},\left\|\left(y^{(k)}, z^{(k)}\right)\right\| \rightarrow \infty$, and $\lambda_{k} \rightarrow \lambda^{*}$ as $k \rightarrow \infty$. From homotopy equation (1), we have

$$
\begin{aligned}
& \left(1-\lambda_{k}\right)\left(x^{(k)}-\Phi\left(x^{(k)}\right)\right)+\sum_{i=1}^{m} \eta_{i}\left(x^{(k)},\left(1-\lambda_{k}\right) y_{i}^{(k)}\right) \\
& +\sum_{j=1}^{l} \zeta_{j}\left(x^{(k)},\left(1-\lambda_{k}\right) z_{j}^{(k)}\right)+\lambda_{k}\left(x^{(k)}-x^{(0)}\right)=0, \\
& h\left(x^{(k)}\right)-\lambda_{k} z^{(k)}=0, \\
& y_{i}^{(k)}\left(g_{i}\left(A_{i}\left(\lambda_{k}\right) x^{(k)}+b_{i}\left(\lambda_{k}\right)\right)+\lambda_{k} c_{i}\right) \\
& -\lambda y_{i}^{(0)}\left(g_{i}\left(A(1) x^{(0)}+b_{i}(1)\right)+c_{i}\right)=0, \\
& \quad i=1, \ldots, m .
\end{aligned}
$$

Let

$$
\begin{aligned}
& I\left(x^{*}\right)=\left\{i \in\{1, \ldots, m\}: \lim _{k \rightarrow \infty} y_{i}^{(k)}=\infty\right\}, \\
& J\left(x^{*}\right)=\left\{j \in\{1, \ldots, l\}: \lim _{k \rightarrow \infty} z_{j}^{(k)}=\infty\right\} .
\end{aligned}
$$

From (19), we have

$$
\begin{aligned}
& g_{i}\left(A_{i}\left(\lambda_{k}\right) x^{(k)}+b_{i}\left(\lambda_{k}\right)\right)+\lambda_{k} c_{i} \\
&=-\lambda_{k}\left(y_{i}^{(k)}\right)^{-1} y_{i}^{(0)}\left(g_{i}\left(A_{i}(1) x^{(0)}+b_{i}(1)\right)+c_{i}\right), \\
& i=1, \ldots, m .
\end{aligned}
$$


If $J\left(x^{*}\right) \neq \emptyset$, rewrite $(17)$ as

$$
\begin{aligned}
& \left(1-\lambda_{k}\right)\left(x^{(k)}-\Phi\left(x^{(k)}\right)\right) \\
& \quad+\sum_{i \notin I\left(x^{*}\right)} \eta_{i}\left(x^{(k)},\left(1-\lambda_{k}\right) y_{i}^{(k)}\right)+\lambda_{k}\left(x^{(k)}-x^{(0)}\right) \\
& \quad+\sum_{j=1}^{l} \zeta_{j}\left(x^{(k)},\left(1-\lambda_{k}\right) z_{j}^{(k)}\right) \\
& \quad+\sum_{i \in I\left(x^{*}\right)} \eta_{i}\left(x^{(k)},\left(1-\lambda_{k}\right) y_{i}^{(k)}\right)=0 .
\end{aligned}
$$

By assumption $\left(A_{2}\right)$, the previous three parts in the left-hand side of (22) are bounded, but the other two parts tend to infinity as $k \rightarrow \infty$; this is impossible. Now we can assume that $z^{(k)} \rightarrow z^{*}$. At the same time we have $I\left(x^{*}\right) \neq \emptyset$. From (21), it is easy to show that $x^{*} \in \partial \Omega_{g}(\lambda)$.

(1) If $\lambda^{*}=1$, from (17), when $k \rightarrow \infty$, then $\left(1-\lambda_{k}\right) y_{i}^{(k)} \rightarrow$ $0, i \notin I\left(x^{*}, 1\right)$, and $\left(1-\lambda_{k}\right) y_{i}^{(k)} \rightarrow y_{i}^{*}, i \in I\left(x^{*}, 1\right)$. Therefore

$$
\begin{aligned}
x^{(0)}= & x^{*}+\lim _{k \rightarrow \infty}\left(1-\lambda_{k}\right)\left(x^{(k)}-\Phi\left(x^{(k)}\right)\right) \\
& +\lim _{k \rightarrow \infty} \sum_{i \in I\left(x^{*}, 1\right)} \eta_{i}\left(x^{(k)},\left(1-\lambda_{k}\right) y_{i}^{(k)}\right) \\
& +\lim _{k \rightarrow \infty} \sum_{i \notin I\left(x^{*}, 1\right)} \eta_{i}\left(x^{(k)},\left(1-\lambda_{k}\right) y_{i}^{(k)}\right) \\
& +\lim _{k \rightarrow \infty} \sum_{j=1}^{l} \zeta_{j}\left(x^{(k)},\left(1-\lambda_{k}\right) z_{j}^{(k)}\right) \\
= & x^{*}+\sum_{i \in I\left(x^{*}, 1\right)} \eta_{i}\left(x^{*}, y_{i}^{*}\right),
\end{aligned}
$$

which contradicts assumption $\left(A_{4}\right)$.

(2) If $0<\lambda^{*}<1$, rewrite (17) as

$$
\begin{aligned}
& \sum_{i \in I\left(x^{*}, \lambda^{*}\right)} \eta_{i}\left(x^{(k)},\left(1-\lambda_{k}\right) y_{i}^{(k)}\right)+\lambda_{k}\left(x^{(k)}-x^{(0)}\right) \\
& +\left(1-\lambda_{k}\right)\left(x^{(k)}-\Phi\left(x^{(k)}\right)\right) \\
& =-\sum_{i \notin I\left(x^{*}, \lambda^{*}\right)} \eta_{i}\left(x^{(k)},\left(1-\lambda_{k}\right) y_{i}^{(k)}\right) \\
& \quad-\sum_{j=1}^{l} \zeta_{j}\left(x^{(k)},\left(1-\lambda_{k}\right) z_{j}^{(k)}\right) .
\end{aligned}
$$

When $k \rightarrow \infty$, because $\Omega_{g}(\lambda)$ and $y_{i}^{(k)}, i \notin I\left(x^{*}, \lambda^{*}\right)$, are bounded, then the right-hand side of (24) is bounded. However, if $y_{i}^{(k)} \rightarrow \infty, i \in I\left(x^{*}, \lambda^{*}\right)$, by assumption $\left(A_{2}\right)$, the left-hand side of (24) tends to infinity. This results in a contradiction.

(3) When $\lambda^{*}=0$, because the nonempty index set $I\left(x^{*}\right) \subset$ $B\left(x^{*}\right)$, the proof of (3) is similar to that of (2).

Now we give the main results of this section.
Theorem 7. Let $H$ be defined as in (1), let $g_{i}(x), i=1, \ldots, m$, and $h_{j}(x), j=1, \ldots, l$, be $C^{3}$ functions, let assumptions $\left(A_{1}\right)-\left(A_{4}\right)$ hold, and let $\eta_{i}\left(x, u_{i}\right) \in R^{n}, i=1, \ldots, m$, and $\zeta_{j}\left(x, v_{j}\right) \in R^{n}, j=1, \ldots, l$, be $C^{2}$ functions. Then, for any $C^{2}$ mapping $\Phi(x): R^{n} \rightarrow R^{n}$ satisfying $\Phi(\Omega) \subset \Omega, \Phi(x)$ has a fixed point in $\Omega$. In addition, for almost all $x^{(0)} \in \Omega_{g}^{0}(1)$, homotopy equation (1) determines a smooth curve $\Gamma_{w^{(0)}}$ starting from $\left(w^{(0)}, 1\right)$. The limit set $T \subset \Omega \times R_{+}^{m} \times R^{l} \times\{0\}$ of $\Gamma_{w^{(0)}}$ is nonempty; and $x$-component of any point in $T$ is a fixed point of $\Phi(x)$ in $\Omega$.

Proof. By Lemma 5, for almost all $x^{(0)} \in \Omega_{g}^{0}(1)$, there exists a smooth curve $\Gamma_{w^{(0)}}$ starting from $\left(w^{(0)}, 1\right)$. Because $\partial H_{w^{(0)}}\left(w^{(0)}, 1\right) / \partial w$ is nonsingular, by the classification theorem of one-dimensional smooth manifold, $\Gamma_{w^{(0)}}$ is diffeomorphic to a unit interval.

Let $\left(w^{*}, \lambda^{*}\right)=\left(x^{*}, y^{*}, z^{*}, \lambda^{*}\right)$. Then the following three cases are possible:
(a) $\left(w^{*}, \lambda^{*}\right)=\left(x^{*}, y^{*}, z^{*}, \lambda^{*}\right) \in \Omega_{g} \times R_{+}^{m} \times R^{l} \times\{0\}$.
(b) $\left(w^{*}, \lambda^{*}\right)=\left(x^{*}, y^{*}, z^{*}, \lambda^{*}\right) \in \Omega_{g}^{0}(1) \times R_{++}^{m} \times R^{l} \times\{1\}$.
(c) $\left(w^{*}, \lambda^{*}\right)=\left(x^{*}, y^{*}, z^{*}, \lambda^{*}\right) \in \partial\left(\Omega_{g}\left(\lambda^{*}\right) \times R_{+}^{m} \times R^{l}\right) \times$ $(0,1]$.

Because $H_{w^{(0)}}(w, 1)=0$ has a unique solution $w^{(0)}$ in $\Omega_{g}^{0}(1) \times$ $R_{++}^{m} \times R^{l} \times\{1\}$, so case (b) is impossible.

In case (c), first, we prove that $y^{*} \notin \partial R_{+}^{m}$. If $y^{*} \in \partial R_{+}^{m}$, then there exist $i_{0} \in\{1, \ldots, m\}$ and a sequence of points $\left\{\left(w^{(k)}, \lambda_{k}\right)\right\} \subset \Gamma_{w^{(0)}}$ such that $y_{i_{0}}^{(k)} \rightarrow y_{i_{0}}^{*}=0$ as $k \rightarrow+\infty$. From the third equation in (1), we have

$$
\begin{aligned}
y_{i_{0}}^{(k)} & \left(g_{i_{0}}\left(A_{i_{0}}\left(\lambda_{k}\right) x^{(k)}+b_{i_{0}}\left(\lambda_{k}\right)\right)+\lambda_{k} c_{i_{0}}\right) \\
= & \lambda_{k} y_{i_{0}}^{(0)}\left(g_{i_{0}}\left(A_{i_{0}}(1) x^{(0)}+b_{i_{0}}(1)\right)+c_{i_{0}}\right) .
\end{aligned}
$$

When $k \rightarrow+\infty$, because $\Omega_{g}(\lambda)$ and $(0,1]$ are bounded, the left-hand side of (25) tends to 0 . At the same time, the righthand side of (25) tends to $\lambda^{*} y_{i_{0}}^{(0)}\left(g_{i_{0}}\left(A_{i_{0}}(1) x^{(0)}+b_{i_{0}}(1)\right)+c_{i_{0}}\right)$, which is strictly less than 0 . This results in a contradiction.

Then we prove that $x^{*} \notin \partial \Omega_{g}(\lambda)$. If $x^{*} \in \partial \Omega_{g}(\lambda)$, then there exist $j_{0} \in\{1, \ldots, m\}$ and a sequence of points $\left\{\left(w^{(k)}, \lambda_{k}\right)\right\} \subset \Gamma_{w^{(0)}}$ such that $g_{j_{0}}\left(A_{j_{0}}(\lambda) x^{(0)}+b_{j_{0}}(\lambda)\right)+\lambda c_{j_{0}} \rightarrow$ $0 ; y_{j_{0}}^{(k)} \rightarrow \infty$ as $k \rightarrow+\infty$. This contradicts Lemma 6, so case (c) is also impossible.

From the above discussion, we obtain that case (a) is the unique possible case. When $\lambda \rightarrow 0$, from (1), $w^{*}$ is a solution of the equation

$$
\begin{aligned}
x-\Phi(x)+\sum_{i=1}^{m} \eta_{i}\left(x, y_{i}\right)+\sum_{j=1}^{l} \zeta_{j}\left(x, z_{j}\right) & =0, \\
h(x) & =0, \\
Y g(x) & =0, \\
g(x) & \leq 0, \quad y \geq 0 .
\end{aligned}
$$


When $x^{*} \in \Omega_{g}^{0}$, we have $g\left(x^{*}\right)<0$ and $h\left(x^{*}\right)=0$, so $x^{*} \in \Omega^{0}$. From the third equation in (26), we obtain $y^{*}=0$. Then the first equation in (26) yields

$$
x^{*}+\sum_{j=1}^{l} \zeta_{j}\left(x^{*}, z_{j}^{*}\right)=\Phi\left(x^{*}\right) .
$$

It follows from assumption $\left(A_{3}\right)$ and $\Phi\left(x^{*}\right) \in X$ that $\sum_{j=1}^{l} \zeta_{j}\left(x^{*}, z_{j}^{*}\right)=0$. Then we easily show that $x^{*}=\Phi\left(x^{*}\right)$ by using assumption $\left(A_{3}\right)$.

When $x^{*} \in \partial \Omega_{g}$, we have $g_{i}\left(x^{*}\right)<0, i \notin B\left(x^{*}\right), g_{i}\left(x^{*}\right)=$ $0, i \in B\left(x^{*}\right)$, and $h\left(x^{*}\right)=0$, so $x^{*} \in \partial \Omega$. Because $g_{i}\left(x^{*}\right)<0$, $i \notin B\left(x^{*}\right)$, from the third equation in (26), $y_{i}^{*}=0, \forall i \notin B\left(x^{*}\right)$. Then (26) yields

$$
x^{*}+\sum_{i \in B\left(x^{*}\right)} \eta_{i}\left(x^{*}, y_{i}^{*}\right)+\sum_{j=1}^{l} \zeta_{j}\left(x^{*}, z_{j}^{*}\right)=\Phi\left(x^{*}\right) .
$$

It follows from assumption $\left(A_{4}\right)$ and $\Phi\left(x^{*}\right) \in \Omega$ that

$$
\sum_{i \in B\left(x^{*}\right)} \eta_{i}\left(x^{*}, y_{i}^{*}\right)+\sum_{j=1}^{l} \zeta_{j}\left(x^{*}, z_{j}^{*}\right)=0 .
$$

By using assumption $\left(A_{3}\right)$, one obtains $z^{*}=0$ and $y_{i}^{*}=0$ for $i \in B\left(x^{*}\right)$, and thus $x^{*}=\Phi\left(x^{*}\right)$. This completes the proof.

\section{Numerical Results}

For almost all $w^{(0)} \in \Omega_{g}^{0}(1) \times R_{++}^{m} \times R^{l}$, by Theorem 7 , the homotopy generates a $C^{1}$ curve $\Gamma_{w^{(0)}}$ by differentiating the first equation of (13); we get the following theorem.

Theorem 8. The homotopy path $\Gamma_{w^{(0)}}$ is determined by the following initial value problem to the ordinary differential equation

$$
\begin{aligned}
D H_{w^{(0)}}(w(s), \lambda(s))\left(\begin{array}{c}
\dot{w}(s) \\
\dot{\lambda}(s)
\end{array}\right) & =0, \\
(w(0), \lambda(0)) & =\left(w^{(0)}, 1\right),
\end{aligned}
$$

where $s$ is the arc length of the curve $\Gamma_{w^{(0)}}$.

Remark 9. Let $A=D H_{w^{(0)}}(w(s), \lambda(s)), v=\left(\dot{w}(s)^{T}, \dot{\lambda}(s)\right)^{T}$, and $y(s)=(w(s), \lambda(s))$. Thus, (30) can be rewritten as

$$
\begin{gathered}
A v=0, \\
y(0)=\left(w^{(0)}, 1\right) .
\end{gathered}
$$

By solving the equation $A v=0$ in (31), we obtain a solution $v$. Thus, (30) becomes the following initial value problem:

$$
\begin{gathered}
\frac{d y}{d s}=v, \\
y(0)=\left(w^{(0)}, 1\right) .
\end{gathered}
$$

By Theorems 7 and 8, for almost all $w^{(0)} \in \Omega_{g}^{0}(1) \times R_{++}^{m} \times R^{l}$, the solution curve $\Gamma_{w^{(0)}}$ of (30) exists. Because the initial value problem (32) is equivalent to (30), the solution of (32) also exists for every $s$.

Now, we discuss how to solve the initial value problem (32). Generally, if we use the numerical methods developed to address the initial value problems of ordinary differential equations to solve (32) alone, then the step length must be sufficiently small to ensure that the sequence $\left\{\left(w^{(k)}, \lambda_{k}\right)\right\}$ is close enough to the solution curve. Using such methods may greatly increase the computational cost. Because the goal is to try to find a point $(w, \lambda)$ (when $\lambda$ is approximately zero) instead of tracking the smooth curve $\Gamma_{w^{(0)}}$ very precisely, it is desirable to combine the numerical methods for solving initial value problems of ordinary differential equations with other methods to develop more efficient methods that are later called predictor-corrector methods [11].

Below, we describe the implementation of a standard predictor-corrector method in detail. Suppose that we have obtained a sequence of points $\left(w^{(i)}, \lambda_{i}\right), i=1, \ldots, k$, starting with an initial guess $\left(w^{(0)}, 1\right)$. To obtain $\left(w^{(k+1)}, \lambda_{k+1}\right)$, we must compute the tangent vector $v$. By the basic theory of homotopy methods, the vector $v$ satisfies the following conditions:

(a) $\|v\|=1$.

(b) $v$ keeps the sign of the determinant of $\left(A^{T}, v\right)^{T}$ invariant.

We then consider the following system:

$$
A v=0, \quad\|v\|=1
$$

which can enable us to obtain a unit tangent vector $v^{(k)}$ at $\left(w^{(k)}, \lambda_{k}\right)$. One of the main solution strategies for (33) is based on the $\mathrm{QR}$ decomposition. For more details, the reader is referred to [11]. By Theorem 7, the matrix $A \in$ $R^{(n+m+l) \times(n+m+l+1)}$ is of full row rank. From (33), the unit tangent vector $v$ at a point on $\Gamma_{w^{(0)}}$ has two opposite directions: one (the positive direction) makes $s$ increase, and the other (the negative direction) makes $s$ decrease. Because the negative direction will lead us back to the initial point, we must go along the positive direction. In the following discussion, for convenience, $A$ is denoted by $D H_{w^{(0)}}(w, \lambda)$. The criterion that determines the positive direction is based on condition (b). In the first iteration, the sign is determined by the following lemma.

Lemma 10. If $\Gamma_{w^{(0)}}$ is smooth, then the positive direction $v^{(0)}$ at the initial guess $\left(w^{(0)}, 1\right)$ satisfies

$$
\operatorname{sign}\left|\begin{array}{c}
D H_{w^{(0)}}\left(w^{(0)}, 1\right) \\
v^{(0)^{T}}
\end{array}\right|=(-1)^{m+l+1}
$$


Proof. Because

$$
\begin{aligned}
D H_{w^{(0)}}\left(w^{(0)}, 1\right) & =\frac{\partial H_{w^{(0)}}\left(w^{(0)}, 1\right)}{\partial(w, \lambda)} \\
& =\left(\begin{array}{cccc}
I & 0 & 0 & a^{(0)} \\
\nabla h\left(x^{(0)}\right)^{T} & 0 & -I & -h\left(x^{(0)}\right) \\
A & B & 0 & b^{(0)}
\end{array}\right) \\
& =\left(M_{1}, M_{2}\right),
\end{aligned}
$$

where

$$
\begin{aligned}
a= & -(x-\Phi(x))-\sum_{i=1}^{m} \frac{\partial \eta_{i}\left(x,(1-\lambda) y_{i}\right)}{\partial \lambda} \\
& -\sum_{j=1}^{l} \frac{\partial \zeta_{j}\left(x,(1-\lambda) z_{j}\right)}{\partial \lambda}, \\
a^{(0)}= & \left.a\right|_{w=w^{(0)}, \lambda=1}, \\
b_{i}= & \frac{\partial\left(y_{i}\left(g_{i}\left(A_{i}(\lambda) x+b_{i}(\lambda)\right)+\lambda c_{i}\right)\right)}{\partial \lambda} \\
& -y_{i}^{(0)}\left(g_{i}\left(A(1) x^{(0)}+b_{i}(1)\right)+c_{i}\right), \\
b^{(0)}= & \left.\left(b_{1}, \ldots, b_{m}\right)^{T}\right|_{w=w^{(0)}, \lambda=1}, \\
M_{1} \in & R^{(n+m+l) \times(n+m+l)}, \\
M_{2} \in & R^{(n+m+l) \times 1},
\end{aligned}
$$

the tangent vector $v^{(0)}$ at $\left(w^{(0)}, 1\right)$ satisfies

$$
\left(M_{1}, M_{2}\right) v^{(0)}=\left(M_{1}, M_{2}\right)\left(\begin{array}{c}
v_{1}^{(0)} \\
v_{2}^{(0)}
\end{array}\right)=0
$$

where $v_{1}^{(0)} \in R^{n+m+l}$ and $v_{2}^{(0)} \in R^{1}$. From (37), it is easy to show that $v_{1}^{(0)}=-M_{1}^{-1} M_{2} v_{2}^{(0)}$. Therefore, the determinant of $\left(\begin{array}{c}D H_{w^{(0)}}\left(w^{(0)}, 1\right) \\ v^{(0)^{T}}\end{array}\right)$ is

$$
\begin{aligned}
& \left|\begin{array}{c}
D H_{w^{(0)}}\left(w^{(0)}, 1\right) \\
v^{(0)^{T}}
\end{array}\right|=\left|\begin{array}{cc}
M_{1} & M_{2} \\
v_{1}^{(0)^{T}} & v_{2}^{(0) T}
\end{array}\right| \\
& =\left|\begin{array}{cc}
M_{1} & M_{2} \\
-M_{2}^{T} M_{1}^{-T} & 1
\end{array}\right| v_{2}^{(0)}=\left|M_{1}\right| v_{2}^{(0)}(1
\end{aligned}
$$

$$
\begin{aligned}
& \left.+M_{2}^{T} M_{1}^{-T} M_{1}^{-1} M_{2}\right)=\left|\begin{array}{ccc}
I & 0 & 0 \\
\nabla h\left(x^{(0)}\right)^{T} & 0 & -I \\
A & B & 0
\end{array}\right| v_{2}^{(0)}(1 \\
& \left.+M_{2}^{T} M_{1}^{-T} M_{1}^{-1} M_{2}\right)=|-I||B| v_{2}^{(0)}(1 \\
& \left.+M_{2}^{T} M_{1}^{-T} M_{1}^{-1} M_{2}\right)=(-1)^{l} \\
& \cdot \mid \operatorname{diag}\left(g_{1}\left(A_{1}(1) x^{(0)}+b_{1}(1)\right)\right. \\
& \left.+c_{1}, \ldots, g_{m}\left(A_{m}(1) x^{(0)}+b_{m}(1)\right)+c_{m}\right) \mid \cdot v_{2}^{(0)}(1 \\
& \left.+M_{2}^{T} M_{1}^{-T} M_{1}^{-1} M_{2}\right) .
\end{aligned}
$$

Because $g_{i}\left(A_{i}(1) x^{(0)}+b_{i}(1)\right)+c_{i}<0, i=1, \ldots, m$, $\left(1+M_{2}^{T} M_{1}^{-T} M_{1}^{-1} M_{2}\right)>0$ and $v_{2}^{(0)}>0$, the sign of the determinant $\left|\begin{array}{c}D H_{w^{(0)}\left(w^{(0)}, 1\right)}^{(0)^{T}} \\ v^{(1)}\end{array}\right|$ is $(-1)^{m+l+1}$.

Therefore, by using the Euler method, for some small step length $h_{k}>0$ (not too small), we are able to obtain a predictor point $\left(\bar{w}^{(k)}, \bar{\lambda}_{k}\right)=\left(w^{(k)}, \lambda_{k}\right)+h_{k} v^{(k)}$. Here, we do not substitute more complicated algorithms for the Euler method because the predictor point does not need to be close enough to the smooth curve $\Gamma_{w^{(0)}}$ if only it lies in the interior of the convergence domain of Newton's method during the corrector phase.

Next, we may perform a corrector step. Set

$$
\begin{aligned}
& D H_{w^{(0)}}(w, \lambda)^{+} \\
& \quad=D H_{w^{(0)}}(w, \lambda)^{T}\left(D H_{w^{(0)}}(w, \lambda) D H_{w^{(0)}}(w, \lambda)^{T}\right)^{-1},
\end{aligned}
$$

which is the Moore-Penrose inverse of $D H_{w^{(0)}}(w, \lambda)$. The corrector phase then tries to identify a corrector point $\left(w^{(k+1)}, \lambda_{k+1}\right)$ on the path $\Gamma_{w^{(0)}}$. The corrector step is usually carried out by Newton's method that uses the Moore-Penrose inverse of $D H_{w^{(0)}}(w, \lambda)$, starting with $\left(\bar{w}^{(k)}, \bar{\lambda}_{k}\right)$ and proceeding until $\left\|H_{w^{(0)}}\left(w^{(k+1)}, \lambda_{k+1}\right)\right\|$ is approximately zero. The following pseudocode describes the basic steps of a generic predictor-corrector method.

Algorithm 11 (Euler-Newton method).

Step 0. Provide an initial guess $\left(w^{(0)}, 1\right)$, an initial step length $h_{0}>0$, and three small positive numbers $\epsilon_{1}>0$, $\epsilon_{2}>0$, and $\epsilon_{3}>0$. Set $k=0$.

Step 1. Compute the direction $\eta^{(k)}$ of the predictor step:

(a) Compute a unit tangent vector $v^{(k)}$.

(b) Determine the direction $\eta^{(k)}$ of the predictor step as follows:

If the sign of the determinant $\left|\begin{array}{c}D H_{w^{(0)}}\left(w^{(k)}, \lambda_{k}\right) \\ v^{(k)^{T}}\end{array}\right|$ is $(-1)^{m+l+1}$, then $\eta^{(k)}=v^{(k)}$. 
TABLE 1: Numerical results of Examples 12-14.

\begin{tabular}{ccccccc}
\hline Example & $x^{(0)}$ & IT & $\lambda^{*}$ & $H$ & $x^{*}$ & $\Phi\left(x^{*}\right)$ \\
\hline 2 & $(-0.5,0.3)$ & 12 & 0.000000 & 0.000000 & $(-0.977199,0.000000)$ & $(-0.977199,0.000000)$ \\
& $(-0.5,-0.3)$ & 13 & 0.000000 & 0.000000 & $(-0.977377,0.000000)$ & $(-0.977377,0.000000)$ \\
\hline \multirow{2}{*}{3} & $(4,2)$ & 9 & 0.000000 & 0.000000 & $(3.000000,0.000000)$ & $(3.000000,0.000000)$ \\
& $(4,-2)$ & 7 & 0.000000 & 0.000000 & $(3.000000,-0.000001)$ & $(3.000000,-0.000001)$ \\
\hline \multirow{2}{*}{4} & $(-3,1)$ & 10 & 0.000000 & 0.000000 & $(-0.000000,-2.500000)$ & $(-0.000000,-2.500000)$ \\
& $(3,1)$ & 10 & 0.000000 & 0.000000 & $(0.000000,-2.500001)$ & $(0.000000,-2.500001)$ \\
\hline
\end{tabular}

If the sign of the determinant $\left|\begin{array}{c}D H_{w^{(0)}}\left(w^{(k)}, \lambda_{k}\right) \\ v^{(k)^{T}}\end{array}\right|$ is
$(-1)^{m+l}$, then $\eta^{(k)}=-v^{(k)}$.

Step 2. Compute a corrector point $\left(w^{(k+1)}, \lambda_{k+1}\right)$.

$$
\begin{aligned}
& \left(\bar{w}^{(k)}, \bar{\lambda}_{k}\right)=\left(w^{(k)}, \lambda_{k}\right)+h_{k} \eta^{(k)}, \\
& \left(w^{(k+1)}, \lambda_{k+1}\right) \\
& \quad=\left(\bar{w}^{(k)}, \bar{\lambda}_{k}\right)-D H_{w^{(0)}}\left(\bar{w}^{(k)}, \bar{\lambda}_{k}\right)^{+} H_{w^{(0)}}\left(\bar{w}^{(k)}, \bar{\lambda}_{k}\right) .
\end{aligned}
$$

If $\left\|H_{w^{(0)}}\left(w^{(k+1)}, \lambda_{k+1}\right)\right\| \leq \epsilon_{1}$, then let $h_{k+1}=$ $\min \left\{h_{0}, 2 h_{k}\right\}$, and go to Step 3 .

If $\left\|H_{w^{(0)}}\left(w^{(k+1)}, \lambda_{k+1}\right)\right\| \in\left(\epsilon_{1}, \epsilon_{2}\right)$, then let $h_{k+1}=h_{k}$, and go to Step 3.

If $\left\|H_{w^{(0)}}\left(w^{(k+1)}, \lambda_{k+1}\right)\right\| \geq \epsilon_{2}$, then let $h_{k+1}=$ $\max \left\{2^{-25} h_{0},\left(h_{k} / 2\right)\right\}, k=k+1$, and go to Step 2 .

Step 3. If $\lambda_{k+1} \leq \epsilon_{3}$, then stop. Otherwise, $k=k+1$, and go to Step 1.

By using homotopy (1) and the predictor-corrector algorithm, we give several numerical examples to illustrate the work in this paper. In each example, we set $\epsilon_{1}=1 \cdot e-3$, $\epsilon_{2}=1 \cdot e-6$, and $h_{0}=0.02$. The behaviors of homotopy paths are shown in Figures 3, 4, and 5. Computational results are given in Table 1 , where $x^{(0)}$ denotes the initial guess, IT denotes the number of iterations, $H$ denotes the value of $\left\|H_{w^{(0)}}\left(w^{(k)}, \lambda_{k}\right)\right\|$ when the algorithm stops, and $x^{*}$ denotes the fixed point.

Example 12 (see Example 1). In this example, we select two initial points $x_{1}^{(0)}=(-0.5,0.3)$ and $x_{2}^{(0)}=(-0.5,-0.3)$ in $\Omega_{g}^{0}(1)$. The pathways generated by homotopy equation (1) are shown in Figure 3. When $\lambda \rightarrow 0$, we can find a fixed point $x^{*}=(-0.977199,0)$ of $\Phi(x)$ in $\Omega_{g}$ following the homotopy pathways $\Gamma_{w^{(0)}}^{1}$ and $\Gamma_{w^{(0)}}^{2}$, respectively.

Example 13. To find a fixed point of self-mapping $\Phi(x)=$ $\left(x_{1},-x_{2}\right)^{T}$ in

$$
\begin{aligned}
\Omega & =\left\{\left(x_{1}, x_{2}\right) \in R^{2}: 3 x_{1}+2 x_{2}^{2}-9=0,-4 x_{2}-13\right. \\
& \left.\leq 0,4 x_{2}-13 \leq 0\right\},
\end{aligned}
$$

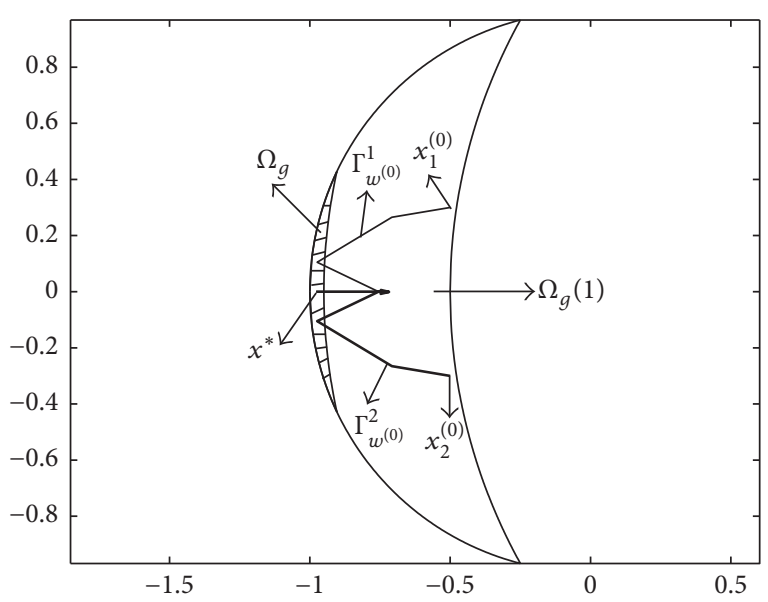

FIGURE 3: The pathways generated by homotopy (1).

in this example, we introduce the $C^{2}$ functions $\xi(x, y)=$ $\nabla g(x) y$ and $\zeta(x, z)=(-8,0)^{T} z$. Assumptions $\left(A_{1}\right)-\left(A_{4}\right)$ are easily verified. We set $\Omega_{g}^{0}=\left\{\left(x_{1}, x_{2}\right) \in R^{2}:-4 x_{2}-\right.$ $\left.13<0,4 x_{2}-13<0\right\}$ and select two initial points $x_{3}^{(0)}=$ $(4,2)$ and $x_{4}^{(0)}=(4,-2)$ in $\Omega_{g}^{0}$. The initial points can be more easily selected in $\Omega_{g}^{0}$ than in $\Omega^{0}$, because $\Omega^{0}$ contains the equality constraint $3 x_{1}+2 x_{2}^{2}-9=0$. The pathways generated by homotopy equation (1) are shown in Figure 4. When $\lambda \rightarrow 0$, we can find a fixed point $x^{*}=(3,0)$ of $\Phi(x)$ in $\Omega$ following the homotopy pathways $\Gamma_{w^{(0)}}^{3}$ and $\Gamma_{w^{(0)}}^{4}$, respectively.

Example 14. To find a fixed point of self-mapping $\Phi(x)=$ $\left(-x_{1}, x_{2}\right)^{T}$ in $\Omega=\left\{\left(x_{1}, x_{2}\right) \in R^{2}: 2 x_{2}+3 x_{1}^{2}+5=\right.$ $\left.0,-x_{1}-4.5 \leq 0, x_{1}-4.5 \leq 0\right\}$, In this example, we introduce the $C^{2}$ functions $\xi(x, y)=\nabla g(x) y$ and $\zeta(x, z)=$ $(0,-6)^{T} z$. Assumptions $\left(A_{1}\right)-\left(A_{4}\right)$ are easily verified. We set $\Omega_{g}^{0}=\left\{\left(x_{1}, x_{2}\right) \in R^{2}:-x_{1}-4.5<0, x_{1}-4.5<0\right\}$ and select two initial points $x_{5}^{(0)}=(-3,1)$ and $x_{6}^{(0)}=(3,1)$ in $\Omega_{g}^{0}$. The initial points can be more easily selected in $\Omega_{g}^{0}$ than in $\Omega^{0}$, because $\Omega^{0}$ contains the equality constraint $2 x_{2}+3 x_{1}^{2}+5=0$. The pathways generated by homotopy equation (1) are shown in Figure 5. When $\lambda \rightarrow 0$, we can find a fixed point $x^{*}=$ $(0,-2.5)$ of $\Phi(x)$ in $\Omega$ following the homotopy pathways $\Gamma_{w^{(0)}}^{5}$ and $\Gamma_{w^{(0)}}^{6}$, respectively. 


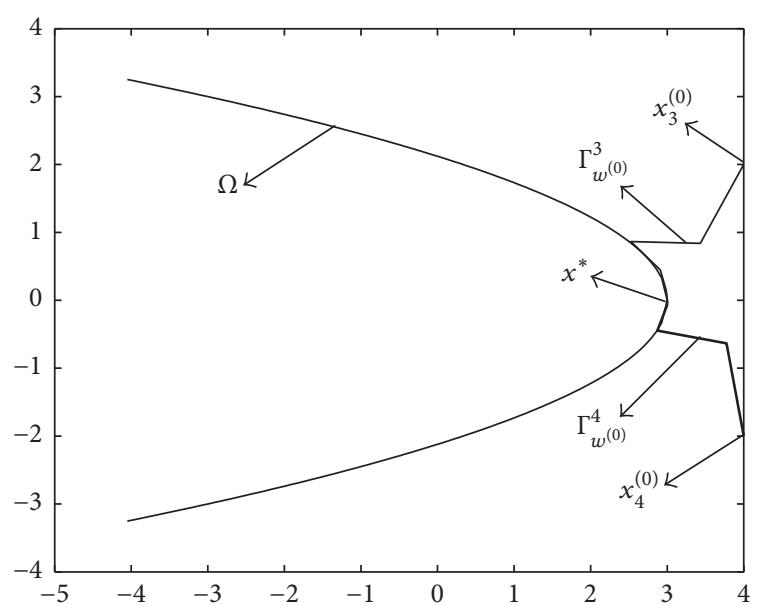

FIgURE 4: The pathways generated by homotopy (1).

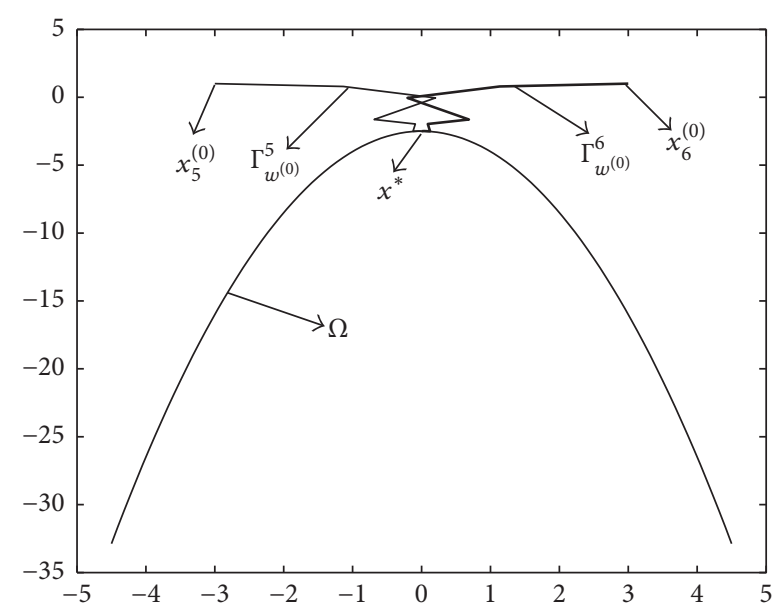

FIgURE 5: The pathways generated by homotopy (1).

\section{Conclusions}

In this paper, we apply appropriate perturbations to the constraint functions and hence develop a parameter perturbation homotopy continuation method for solving fixed point problems with both inequality and equality constraints. Our results make initial points of the algorithm be chosen more easily than the previous results in the literature and improve the efficiency of the algorithm greatly. Because fixed point problems have been widely applied to many areas such as mechanics, physics, transportation, control, economics, differential equations, and optimization, our results may be useful to propose a powerful solution tool for dealing with these practical problems. In the future, we want to propose new techniques to extend our results to more general nonconvex sets and extend the method proposed in this paper to solve the periodicity problems of some important differential equations.

\section{Competing Interests}

The authors declare that there are no competing interests regarding the publication of this paper.

\section{Acknowledgments}

This work was supported by National Natural Science Foundation of China (no. 11671188 and no. U1304103).

\section{References}

[1] B. Bollobas, W. Fulton, A. Katok, F. Kirwan, and P. Sarnak, Fixed Point Theory and Applications, Cambridge University Press, 2004.

[2] S. Heikkila and K. Reffett, "Fixed point theorems and their applications to theory of Nash equilibria," Nonlinear Analysis. Theory, Methods \& Applications, vol. 64, no. 7, pp. 1415-1436, 2006.

[3] L.-J. Lin and Z.-T. Yu, "Fixed-point theorems and equilibrium problems," Nonlinear Analysis. Theory, Methods \& Applications, vol. 43, no. 8, pp. 987-999, 2001.

[4] S. Park, "Fixed points and quasi-equilibrium problems," Mathematical and Computer Modelling, vol. 32, pp. 1297-1303, 2000.

[5] Y. Yao, Y.-C. Liou, and J.-C. Yao, "Convergence theorem for equilibrium problems and fixed point problems of infinite family of nonexpansive mappings," Fixed Point Theory and Applications, Art. ID 64363, 12 pages, 2007.

[6] Y. Yao, R. Chen, and J.-C. Yao, "Strong convergence and certain control conditions for modified Mann iteration," Nonlinear Analysis. Theory, Methods \& Applications, vol. 68, no. 6, pp. 1687-1693, 2008.

[7] Y. Yao, R. Chen, and Y.-C. Liou, "A unified implicit algorithm for solving the triple-hierarchical constrained optimization problem," Mathematical and Computer Modelling, vol. 55, no. 3-4, pp. 1506-1515, 2012.

[8] Y. Yao, Y.-C. Liou, and J.-C. Yao, "Split common fixed point problem for two quasi-pseudo-contractive operators and its algorithm construction," Fixed Point Theory and Applications, vol. 2015, article no. 127, 2015.

[9] Y. Yao, Y.-C. Liou, and J.-C. Yao, "Finding the minimum norm common element of maximal monotone operators and nonexpansive mappings without involving projection," Journal of Nonlinear and Convex Analysis, vol. 16, no. 5, pp. 835-854, 2015.

[10] Y. Yao, M. Postolache, Y.-C. Liou, and Z. Yao, "Construction algorithms for a class of monotone variational inequalities," Optimization Letters, vol. 10, no. 7, pp. 1519-1528, 2016.

[11] E. L. Allgower and K. Georg, Introduction to Numerical Continuation Methods, vol. 45 of Classics in Applied Mathematics, SIAM, Philadelphia, Pa, USA, 2003.

[12] C. B. Garcia and W. I. Zangwill, "An approach to homotopy and degree theory," Mathematics of Operations Research, vol. 4, no. 4, pp. 390-405, 1979.

[13] S. N. Chow, J. Mallet-Paret, and J. A. Yorke, "Finding zeroes of maps: homotopy methods that are constructive with probability one," Mathematics of Computation, vol. 32, no. 143, pp. 887-899, 1978.

[14] R. B. Kellogg, T. Y. Li, and J. Yorke, "A constructive proof of the Brouwer fixed-point theorem and computational results," SIAM Journal on Numerical Analysis, vol. 13, no. 4, pp. 473-483, 1976. 
[15] Y. Li and Z. H. Lin, "A constructive proof of the PoincareBirkhoff theorem," Transactions of the American Mathematical Society, vol. 347, no. 6, pp. 2111-2126, 1995.

[16] B. Yu and Z. Lin, "Homotopy method for a class of nonconvex Brouwer fixed-point problems," Applied Mathematics and Computation, vol. 74, no. 1, pp. 65-77, 1996.

[17] M. Su and Z. Liu, "Modified homotopy methods to solve fixed points of self-mapping in a broader class of nonconvex sets," Applied Numerical Mathematic, vol. 58, no. 3, pp. 236-248, 2008.

[18] Z. Zhu, B. Yu, and Y. Shang, "A modified homotopy method for solving nonconvex fixed points problems," Fixed Point Theory, vol. 14, no. 2, pp. 531-544, 2013.

[19] X. Fan, F. Gao, and T. Xu, "An infeasible homotopy method for solving fixed point problems on a general unbounded set," Journal of Applied Mathematics and Computing, vol. 47, no. 1-2, pp. 277-289, 2015.

[20] Z. Zhu and L. Yang, "A constraint shifting homotopy method for computing fixed points on nonconvex sets," Journal of Nonlinear Science and its Applications. JNSA, vol. 9, no. 6, pp. 3850-3857, 2016.

[21] G. L. Naber, Topological Methods in Euchdean Space, Cambridge University Press, London, UK, 1980. 


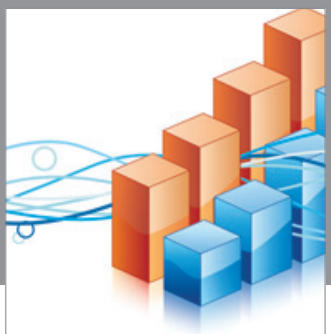

Advances in

Operations Research

vatem alat4

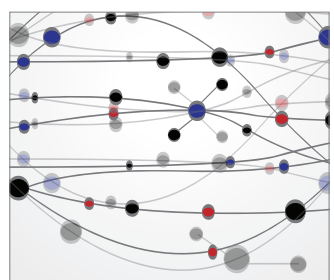

\section{The Scientific} World Journal
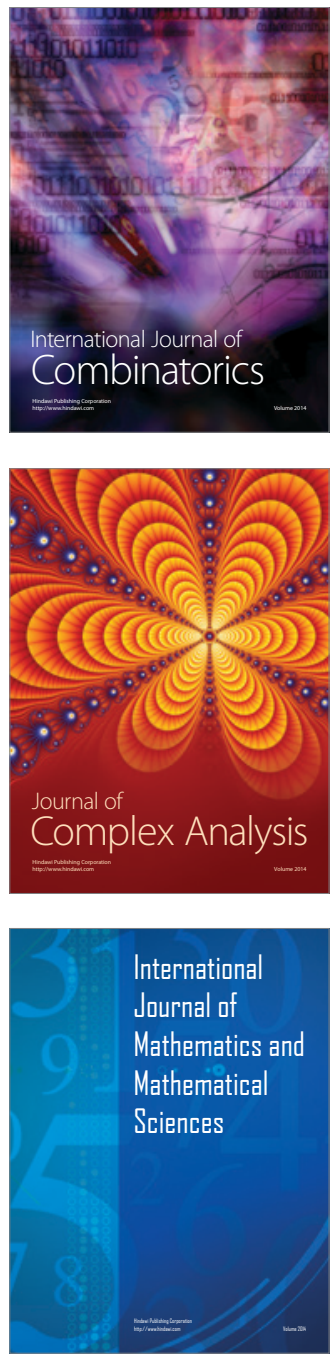
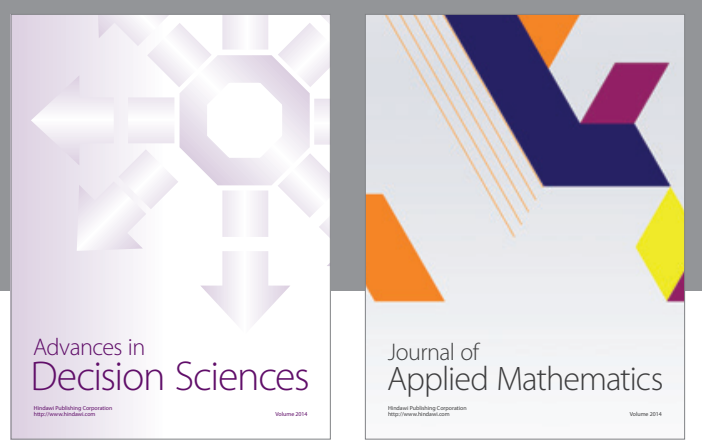

Algebra

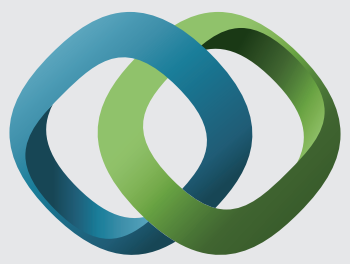

\section{Hindawi}

Submit your manuscripts at

https://www.hindawi.com
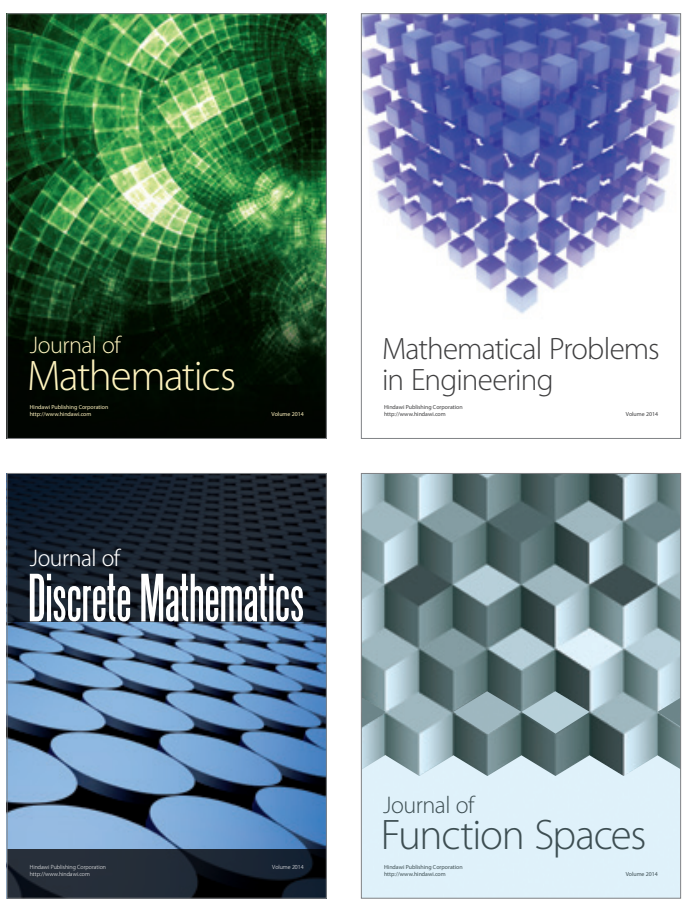

Mathematical Problems in Engineering
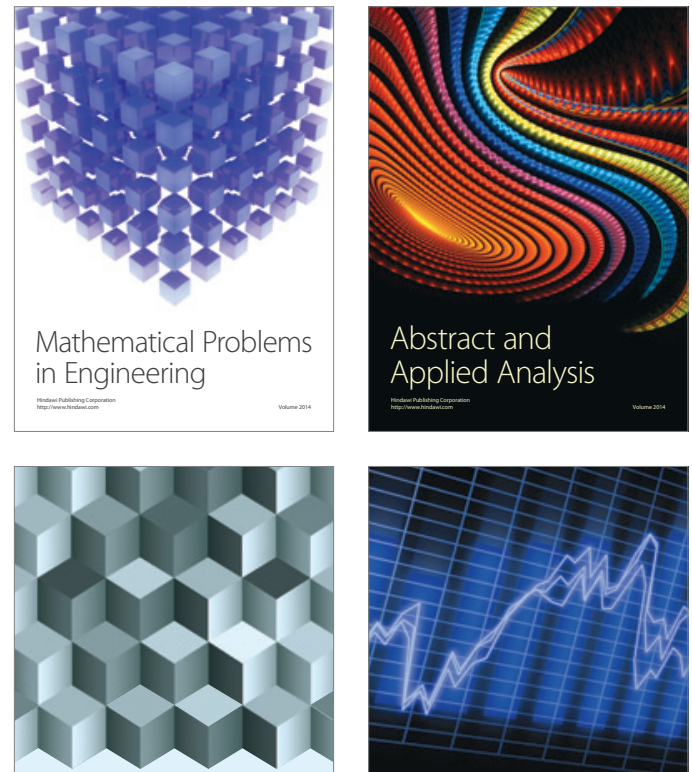

Journal of

Function Spaces

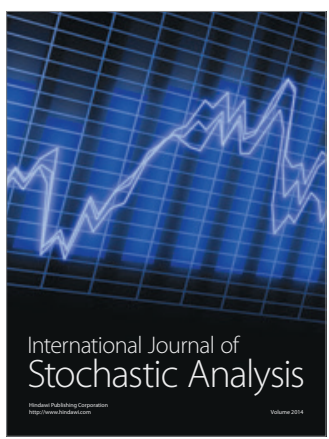

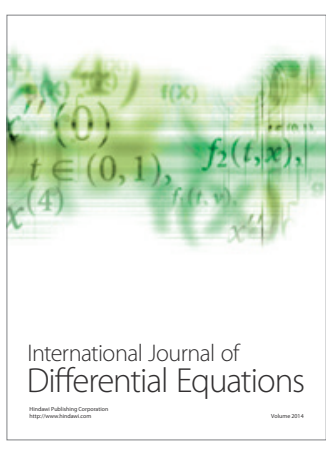
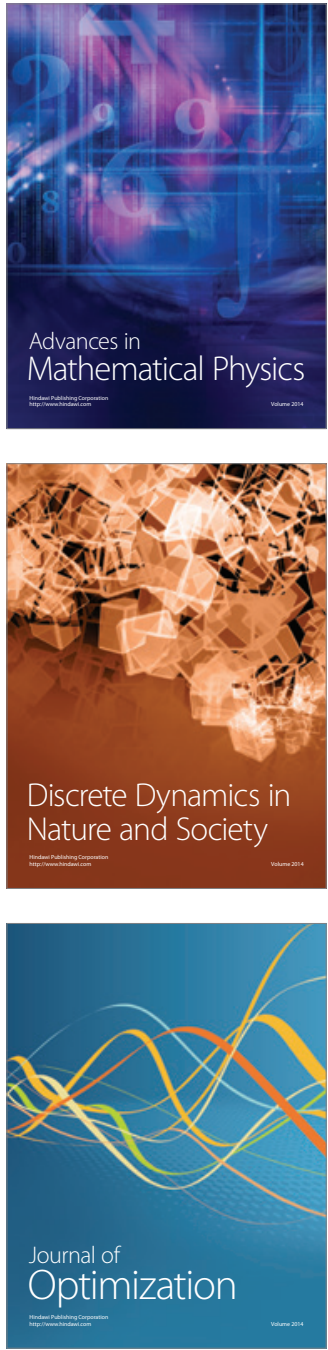